\title{
A Psychometric Evaluation of the Behavioral Inhibition Questionnaire in a Non-Clinical Sample of Dutch Children and Adolescents
}

\author{
Suzanne Broeren • Peter Muris
}

Published online: 18 November 2009

(C) The Author(s) 2009. This article is published with open access at Springerlink.com

\begin{abstract}
The Behavioral Inhibition Questionnaire (BIQ) is a parent-rating scale for measuring temperamental characteristics referring to shyness, fearfulness, and withdrawal in young, preschool children. The present study evaluated the psychometric properties of the BIQ in a Dutch community sample of children with a broad age range. For this purpose, the reliability and validity of the BIQ was evaluated in three age groups: 4-7-year-olds, 8-11-year-olds, and 12-15-year-olds. The results indicated that the internal consistency of most BIQ scales was satisfactory in all three age groups. Principal component analysis of the BIQ yielded a six-factor model that was largely in keeping with the hypothesized structure consisting of the social and non-social components of behavioral inhibition. Confirmatory factor analysis indicated that this model provided a reasonable fit for the data. Further, support for the validity of the measure was obtained in all age groups. That is, BIQ scores were positively correlated with a wide range of anxiety symptoms, although the most substantial links were found for symptoms of social anxiety. Finally, a self-report version of the BIQ, which was administered to children aged 9 years and above, was found to possess good internal consistency and adequate parent-child agreement. Altogether, the results of this study indicate that suggests that the BIQ might be a reliable and valid measure for assessing behavioral inhibition not only in preschoolers but also in older children and adolescents.
\end{abstract}

Keywords Behavioral Inhibition Questionnaire - Temperament · Anxiety · Psychometric properties $\cdot$ Children $\cdot$ Adolescents

\footnotetext{
S. Broeren $(\bowtie)$

Institute of Psychology, Erasmus University Rotterdam, Burgemeester Oudlaan 50, Suite T13-08, P.O. Box 1738, 3000 DR Rotterdam, The Netherlands

e-mail: broeren@fsw.eur.nl

P. Muris

Institute of Psychology, Erasmus University Rotterdam, Burgemeester Oudlaan 50, Suite T13-37, P.O. Box 1738, 3000 DR Rotterdam, The Netherlands

e-mail: muris@fsw.eur.nl
} 
Behavioral inhibition can be defined as a temperament characteristic referring to the tendency to be unusually shy and to respond with fearfulness and withdrawal in new and unfamiliar social and non-social situations [1]. Behavioral inhibition has a genetic basis [2] and about $10-15 \%$ of the children can be categorized as highly inhibited [3]. Research has indicated that behaviorally inhibited children seem to run a higher risk for developing an anxiety disorder. For example, Biederman et al. [4] found in their study on psychiatric correlates of behavioral inhibition in young children of parents with and without psychiatric disorders, that inhibited children more often displayed multiple anxiety disorders than uninhibited children. A 3-year follow-up of this sample revealed that children initially categorized as inhibited showed a marked increase in anxiety problems that was not observed in the uninhibited group [5]. In specific, the rates of multiple anxiety disorders, separation anxiety disorder, and social anxiety disorder had substantially increased.

Although there is evidence indicating that behavioral inhibition should be conceived as a general risk factor that predisposes children to a wide range of anxiety problems, some authors have suggested that behavioral inhibition represents a more specific risk factor that would only be relevant for the development of social anxiety symptoms. For example, Mick and Telch [6] asked 38 undergraduates with high levels of social anxiety and/or generalized anxiety symptoms, and 38 undergraduates with low levels of such symptoms to retrospectively report their levels of behavioral inhibition during childhood. They found that behavioral inhibition was positively linked to social anxiety, but not to generalized anxiety symptoms. Gladstone et al. [7] found further evidence for the relation between childhood behavioral inhibition and social anxiety in a sample of 189 adult patients with major depression. Patients were divided in three groups scoring low, moderate, or high on behavioral inhibition. Patients who retrospectively reported high levels of behavioral inhibition during childhood were more likely to qualify for a diagnosis of social phobia, but not for other anxiety disorder diagnoses, than patients who reported low or moderate levels of behavioral inhibition. Moreover, there is also a study providing longitudinal support for behavioral inhibition being a specific risk factor for social anxiety problems. In their threeyear prospective study among 261 non-clinical children aged 5-8 years, Van Brakel et al. [8] investigated the role of behavioral inhibition as a specific risk factor in the development of a broad range of anxiety disorder symptoms. Results indicated that behavioral inhibition was only predictive of social anxiety symptoms and not for anxiety symptoms in general.

The issue whether behavioral inhibition should be viewed as a specific vulnerability factor of social anxiety or as a more general feature that predisposes children to a broad range of anxiety problems, may have repercussions for the assessment of this temperamental trait. In young children, behavioral inhibition has been typically assessed by means of laboratory procedures in which children are exposed to various types of social (e.g., an unknown peer or adult) as well as non-social (e.g., a black box or a novel computer game) stimuli. During these procedures, behavioral observations (e.g., spontaneous talk, smiling, looking at the experimenter, and response latency) are obtained that are considered to be indicative for the inhibited temperament. These laboratory procedures for measuring behavioral inhibition are quite extensive and time-consuming, and therefore it is important to note that a number of rating scales have been constructed [9-11]. Unfortunately, it seems that these rating scales are somewhat biased to the assessment of the social aspects of the construct of behavioral inhibition (for a more extensive discussion, see [12]), and this could provide a plausible explanation for that fact that behavioral inhibition is generally found to be more strongly related to social anxiety than to other types of anxiety problems.

The recently developed Behavioral Inhibition Questionnaire (BIQ) [13] seems to be an improvement in this regard as this parent-rating scale measures children's levels of 
behavioral inhibition in various social and non-social domains. So far, two studies have been conducted to examine the psychometric properties of the BIQ, and these have generally yielded positive results $[13,14]$. First of all, the BIQ shows good internal consistency (with Cronbach's alphas for the total score and subscales being $>.70$ ). Second, the test-retest stability over a 12-month period appeared to be satisfactory ( $r$ 's ranging from .49 to .79). Third, strong convergence was found between the ratings of mothers and fathers ( $r$ 's in the .70-.80 range), whereas the convergence between parents and teachers was more moderate but still acceptable ( $r$ 's between .43 and .62 ). Fourth, the validity of the scale also proved to be satisfactory. That is, the BIQ correlated in a theoretically meaningful way with subscales of other temperament scales that measure inhibited behavior, as well as with observations of this temperamental characteristic. Further, confirmatory factor analysis yielded support for a model differentiating BIQ items in various social and nonsocial factors, which are nevertheless allowed to correlate as they seem to represent a higher-order behavioral inhibition factor. Finally, the BIQ was found to be positively associated with a broad range of anxiety symptoms, supporting the idea that if behavioral inhibition is assessed by a scale covering social and non-social aspects, it can best be conceived as a general vulnerability factor.

The BIQ was originally developed for measuring behavioral inhibition in children aged 2-6 years, and as such it is not surprising that previous psychometric evaluations have primarily targeted preschool children. However, there are good reasons to believe that this scale may also be relevant for older youths. Behavioral inhibition has been described as an enduring temperament variable that remains relatively stable from (early) childhood to adolescence and even adulthood [10-16]. As behavioral inhibition is considered to be a vulnerability factor to anxiety pathology operating throughout the entire childhood [17], the need for an instrument like the BIQ may go well beyond the preschool age. Therefore the current study examined the psychometric properties of the BIQ in a Dutch community sample of children and adolescents with a broad age range (i.e., 4-15 years). The parents of these youths completed the BIQ as well as a scale for measuring children's anxiety disorder symptoms. Children aged 9 years and above also completed a child version of the BIQ. In this way, the reliability and validity of the BIQ was examined in three age groups: 4-7-year-olds, 8-11-year-olds, and 12-15-year-olds. It was hypothesized that the psychometric properties of the BIQ would be highly comparable in various age groups. Furthermore, we were able to investigate the reliability of the self-report version of the $\mathrm{BIQ}$, as well as its relation to the parent version. Overall, the BIQ was expected to be a reliable scale with a clear-cut factor structure with distinct but related components representing various social and non-social aspects of behavioral inhibition. In addition, we predicted the BIQ to be positively associated with anxiety symptoms. More specifically, it was hypothesized that the social components of the BIQ would be most strongly associated with social anxiety symptoms, whereas the non-social components would be more convincingly linked to other anxiety symptoms.

\section{Method}

Participants

Participants were 531 children aged $4-15$ years $(M=9.60$ years, SD $=3.41 ; 230$ boys and 301 girls) and their primary caregivers (451 mothers, 44 fathers, 26 mothers/fathers, and 1 older sister). Children were divided in three age groups: $4-7$-year-olds $(n=172$; 
$M=5.42$ years, $\quad \mathrm{SD}=1.09 ; 81$ boys and 91 girls $), 8-11$-year-olds $\quad(n=146$; $M=9.44$ years, $\mathrm{SD}=1.13 ; 63$ boys and 83 girls $)$, and $12-15$-year-olds $(n=213$; $M=13.08$ years, $\mathrm{SD}=0.94 ; 86$ boys and 127 girls). Parents of children from three primary schools and two secondary schools in Rotterdam, Nieuwerkerk aan den IJssel, and Goes, The Netherlands, were invited to participate in this study by providing them with an information letter along with a consent form. Approximately $23 \%$ of the parents (540 out of 2363 parents) responded positively to this mailing and completed the set of questionnaires (see below). Nine parents were excluded from the data analysis because their questionnaires contained too many missing values. The majority of the children $(n=514$; $96.8 \%)$, mothers $(n=456 ; 85.9 \%)$, and fathers were from Dutch descent $(n=472$; $88.9 \%$ ). No other information about the socioeconomic background was available, although it should be mentioned that in the Netherlands non-Caucasian ethnicity is generally associated with a lower socioeconomic status. The present sample seemed to represent the population of the schools which participated in this study rather well. One exception was a primary school with a fairly high percentage of migrant children, which were clearly underrepresented in their school sample and the total sample for this study.

\section{Questionnaires}

\section{Behavioral Inhibition Questionnaire}

The Behavioral Inhibition Questionnaire (BIQ) [13] consists of 30 items assessing behavioral inhibition in three domains: social novelty, situational novelty, and physical challenges. Social novelty is represented by 14 items and is measured in 3 contexts: adults (4 items; e.g., "Is very talkative to adult strangers"), peers (6 items; e.g., "Will happily approach a group of unfamiliar children and join their play"), and performance situations (4 items; e.g., "Enjoys being the center of attention"). Situational novelty consists of 12 items and is measured in two contexts, namely preschool/separation (4 items; e.g., "Quickly adjusts to new situations") and unfamiliar situations (8 items; e.g., "Approaches new situations or activities very hesitantly"). The physical challenges domain contains 4 items (e.g., "Is cautious in activities that involve physical challenge"). Items are rated on a 6-point scale ranging from 1 (hardly ever) to 6 (almost always). Sixteen items are reverse scored (e.g., "Enjoys being the center of attention"). After recoding the reverse scored items, total BIQ (range 30-180) and subscale scores can be calculated by summing across relevant items. ${ }^{1}$

\section{Preschool Anxiety Scale-Revised}

The Preschool Anxiety Scale-Revised (PAS-R) [14], which is a modification of the Preschool Anxiety Scale (PAS) [17], is a parent-based questionnaire for measuring anxiety disorder symptoms in younger children. More precisely, the PAS-R includes 30 items representing symptoms of generalized anxiety disorder $(7$ items; e.g., "Has difficulty stopping him/herself from worrying"), social anxiety disorder (7 items; e.g., "Is afraid to go up to a group of children to join their activities"), obsessive-compulsive disorder ( 2 items; e.g., "Becomes distressed by thoughts or images in his/her head"), specific fears

\footnotetext{
${ }^{1}$ Minor changes were made to some BIQ items in order to make them appropriate for the older children in our sample. For instance, the item "Quickly adjusts to new situations (e.g., kindergarten, preschool, childcare)" was changed into "Quickly adjusts to new situations (e.g., a new class or sporting club)".
} 
(i.e., specific phobia; 9 items; e.g., "Is frightened of dogs"), and separation anxiety disorder (5 items; e.g., "Would be upset at sleeping away from home"). Parents are asked to score each item on a 5-point scale, with anchors 1 (not at all true) and 5 (very often true). PAS-R total (range 30-150) and subscale scores can be computed by summing across relevant items.

Psychometric evaluations of the original and revised versions of the PAS have generally yielded positive results. Data have demonstrated that, with exception of the obsessivecompulsive disorder scale (which consists of only 2 items), all scales display moderate to good internal consistency, cross-informant reliability, and test-retest stability [14, 18]. First evidence has also been obtained for the validity of the PAS and the PAS-R. That is, factor analysis indicated that symptoms cluster into components that are nicely in keeping with the anxiety disorders as described in the Diagnostic and Statistical Manual of Mental Disorders (APA; [14], [18], [19]). Further, PAS and PAS-R (sub)scales are substantially and positively correlated with the internalizing problems scale of the Child Behavior Checklist [19], whereas fairly low and often non-significant correlations are observed with the externalizing problems scales $[17,20]$.

\section{Child Version of the $B I Q$}

The child version of the BIQ was identical to the questionnaire that was completed by the parents, except that instructions and items were rephrased in terms of children's perspective (e.g., "I am shy when first meeting new children").

\section{Procedure}

All parents received an information letter, a consent form, and a set of questionnaires which were handed out by their child's teacher. When parents decided to participate, they completed the PAS-R and BIQ at home and returned these materials to the researchers via the teacher. Children aged 9-15 years completed the child version of the BIQ during regular classes at school. In most of the schools, children received a small present (i.e., a toy) or candy in return for their participation in this study. Children in one school were rewarded with playing materials for the entire class (e.g., soft balls, footballs).

\section{Data Analysis}

Exploratory factor analysis was employed because this is the first study examining the structure of the Dutch BIQ. Previous research has examined the structure of this parentrating scale in English-speaking countries, but of course it remains to be seen whether this translation of the BIQ behaves psychometrically in a similar way as the original version. Furthermore, it is also the first time this questionnaire is used with older children and adolescents instead of preschoolers. In addition, confirmatory factor analysis (CFA) was conducted using Analysis of Moment Structures (AMOS; version 16). The estimation method employed was maximum likelihood. CFA was used to estimate how well the proposed model (a six correlated factors model) explained the sample data. Various indices were used to evaluate the goodness-of-fit of this model: the Comparative Fit Index (CFI), the Root Mean Square Error of Approximation (RMSEA), and the chi square per degree of freedom $\left(\chi^{2} / d f\right)[22,23]$. The possible values of the CFI range from 0 to 1 , with bigger values indicating better fit and values exceeding .90 indicating good fit. For the RMSEA 
values $<.08$ indicate an acceptable model fit and for the $\chi^{2} / d f$ statistic values between 2 and 4 indicate sufficient model fit.

\section{Results}

\section{Exploratory Factor Analysis}

Principal component analysis with varimax rotation was conducted on the BIQ data of the total sample and the three age groups separately in order to explore the factor structure of the scale. For all three age groups the factor analysis based on the eigenvalue $>1$ rule, yielded a six-factor solution which seemed to be nicely in keeping with the six intended subscales. In the total sample, this model accounted for $68.28 \%$ of the total variance (eigenvalues for the six factors were 12.64, 2.18, 1.94, 1.41, 1.19, and 1.11), and these figures were comparable in the separate age groups. Table 1 displays the factor loadings of various BIQ items for the total sample and also shows whether items loaded on similar factors in each of the three age groups. As can be seen, items generally clustered in the hypothesized factors as described by Bishop et al. [13] and Edwards [14], except for the items referring to unfamiliar situations, which displayed more convincing loadings on other factors (e.g., preschool/separation and adults). Otherwise, few substantial (i.e., >.40) secondary factor loadings emerged, which was true in all three age groups.

\section{Confirmatory Factor Analysis}

A CFA was carried out to test the fit of the hypothesized six correlated factors model of the BIQ as described for the original version $[13,14]$. The fit indices indicated that this model provided a reasonable fit for the data (i.e., $\chi^{2} / d f=3.25, \mathrm{CFI}=.92$, and RMSEA $=.07$ ). Separate analyses were conducted for the three age groups and yielded comparable fit indices (with $\chi^{2} / d f$ values between 1.74 and 2.34, CFI between .88 and .91, and RMSEA between .07 and .08$)$.

\section{Reliability}

Internal consistency coefficients (Cronbach's alphas) and item-total correlations of the total scale and subscales of the BIQ in each of the three age groups are displayed in Table 2. As can be seen, internal consistencies and item-total correlations were all satisfactory and this appeared true for children of various ages. More precisely, Cronbach's alphas varied between .79 and .96 in 4-7-year-olds, .67 and .95 in 8-11-year-olds, and .73 and .95 in 12-15-year-olds. In a similar vein, item-total correlations ranged between .29 and .84 in 4-7-year-olds, .19 and .87 in 8-11-year-olds, and .38 and .82 in 12-15-year-olds.

A series of 3 (age groups) $\times 2$ (gender) analyses of variance was performed to examine the influence of these demographic variables on various BIQ scales. As shown in Table 2, the three age groups differed significantly on only two BIQ subscales [with $F(2,525) \mathrm{s} \geq$ 4.03, all $p \mathrm{~s}<.05$, partial $\left.\eta^{2} \mathrm{~s}>.02\right]$. Post-hoc comparisons revealed that on the BIQ performance situations subscales, children aged 8 to 11 years scored significantly lower than children aged $12-15$ years $(p<.01)$ and that $4-7$ - and $8-11$-year-olds displayed significantly lower scores on the BIQ physical challenges subscale as compared to 12-15year-olds $(p<.001)$. 


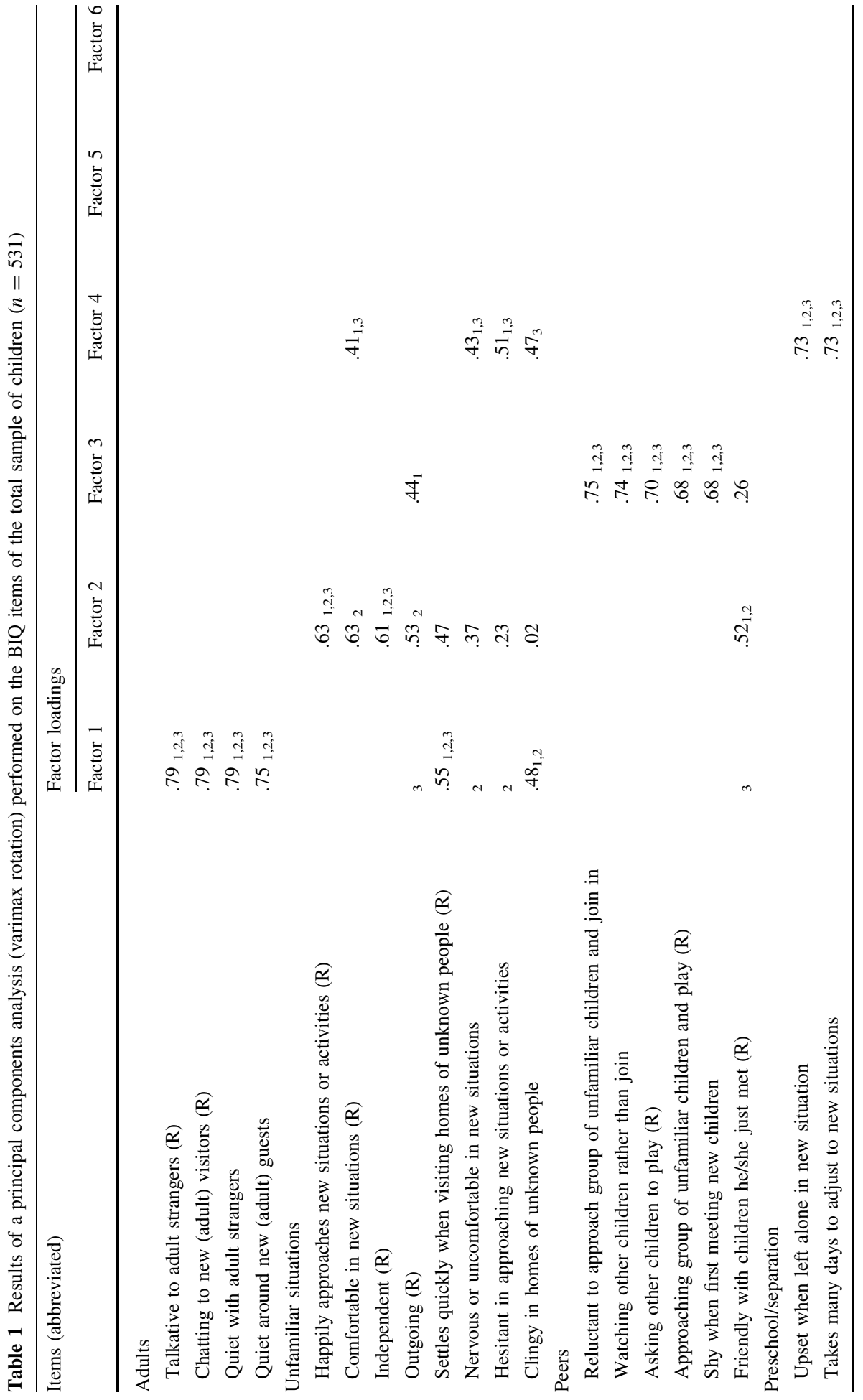




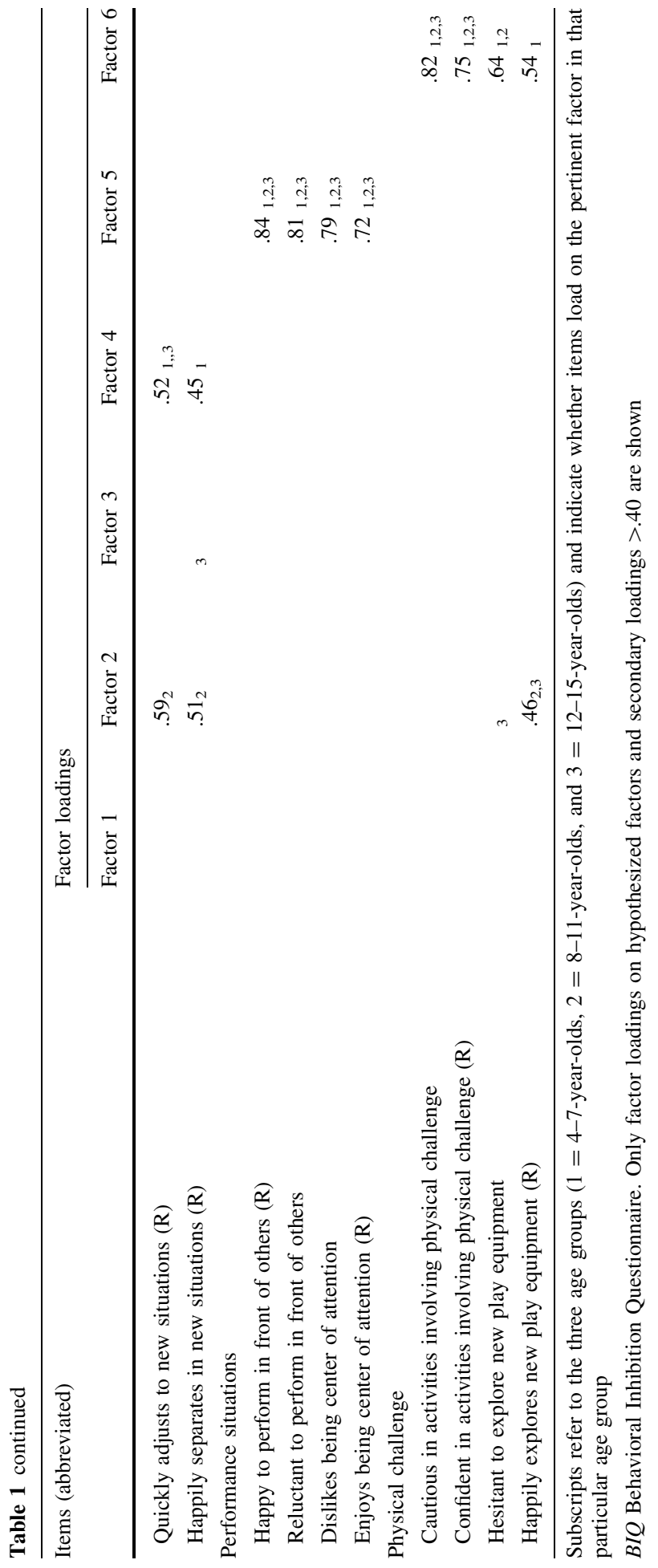


Table 2 Means (standard deviations), Cronbach's alphas, and item-total correlations for various BIQ scales in 4-7-, 8-11- and 12-15-year-olds

\begin{tabular}{|c|c|c|c|c|c|c|c|c|c|}
\hline & \multicolumn{3}{|c|}{ 4-7-year-olds $(n=172)$} & \multicolumn{3}{|c|}{ 8-11-year-olds $(n=146)$} & \multicolumn{3}{|c|}{$12-15$-year-olds $(n=213)$} \\
\hline & $M(\mathrm{SD})$ & $\alpha$ & $\begin{array}{l}\text { Item- } \\
\text { total } r\end{array}$ & $M(\mathrm{SD})$ & $\alpha$ & $\begin{array}{l}\text { Item- } \\
\text { total } r\end{array}$ & $M(\mathrm{SD})$ & $\alpha$ & $\begin{array}{l}\text { Item- } \\
\text { total } r\end{array}$ \\
\hline \multicolumn{10}{|l|}{ BIQ } \\
\hline Total score & $86.72(24.68)$ & .96 & $.29-.83$ & $82.96(24.91)$ & .95 & $.19-.82$ & $87.67(23.91)$ & .95 & $.38-.76$ \\
\hline Peers & $17.91(6.21)$ & .89 & $.48-.81$ & $16.98(5.65)$ & .84 & $.42-.77$ & $18.76(6.17)$ & .87 & $.39-.80$ \\
\hline $\begin{array}{l}\text { Unfamiliar } \\
\text { adults }\end{array}$ & $12.37(5.18)$ & .92 & $.77-.84$ & $11.81(5.14)$ & .92 & $.79-.86$ & $11.65(4.71)$ & .89 & $.73-.81$ \\
\hline $\begin{array}{l}\text { Performance } \\
\text { situations }\end{array}$ & $13.42(4.67) \mathrm{ab}$ & .90 & $.72-.82$ & $12.49(4.91)_{\mathrm{a}}$ & .83 & $.54-.73$ & $14.13(5.18)_{\mathrm{b}}$ & .87 & $.69-.77$ \\
\hline $\begin{array}{l}\text { Preschool/ } \\
\text { separation }\end{array}$ & $10.54(3.89)$ & .84 & $.55-.74$ & $9.87(4.25)$ & .86 & $.61-.79$ & $9.56(3.30)$ & .79 & $.58-.70$ \\
\hline $\begin{array}{l}\text { Unfamiliar } \\
\text { situations }\end{array}$ & $24.03(7.28)$ & .91 & $.57-.83$ & $23.36(7.83)$ & .92 & $.60-.87$ & $23.23(6.95)$ & .89 & $.43-.82$ \\
\hline $\begin{array}{l}\text { Physical } \\
\text { challenges }\end{array}$ & $8.45(3.53)_{\mathrm{a}}$ & .79 & $.53-.73$ & $8.45(3.64)_{a}$ & .67 & $.27-.56$ & $10.22(3.90)_{\mathrm{b}}$ & .73 & $.39-.62$ \\
\hline
\end{tabular}

$B I Q$ Behavioral Inhibition Questionnaire. Means with different subscripts differ after Bonferroni correction at $p<.05$

Further, a significant main effect of gender $[F(1,525)=40.46, p<.001$, partial $\left.\eta^{2}=.07\right]$ and a significant interaction effect of age groups and gender $[F(2,525)=4.27$, $p<.05$, partial $\left.\eta^{2}=.02\right]$ were observed for the BIQ performance situations subscale. Post-hoc comparisons indicated that in 4-7-year-olds $[t(170)=3.61, p<.001$, partial $\left.\eta^{2}=.53\right]$ and $8-11$-year-olds $\left[t(144)=5.80, p<.001\right.$, partial $\left.\eta^{2}=.88\right]$ boys had significantly higher scores on this BIQ subscale than girls, whereas the scores of boys and girls among 12-15-year-olds were not significantly different $[t(211)=1.71, p=.09$, partial $\left.\eta^{2}=.25\right]$.

\section{Validity}

Before discussing the findings concerning the validity of the BIQ, it should be mentioned that all subscales of the PAS-R displayed good internal consistency (Cronbach's alphas $>.70$ ), except for the obsessive-compulsive subscale (Cronbach's alpha $=.47$ which is not surprising given that this subscale only contains two items).

Table 3 shows correlations between the scores on the parent version of the BIQ and the PAS-R. As can be seen, a similar pattern of correlations between BIQ and PAS-R scores emerged for all three age groups. To begin with, the BIQ and PAS-R total scores were substantially correlated (all $r \mathrm{~s}>$.66). Further, significant and positive correlations were generally found between the BIQ subscale scores and scores on all PAS-R subscales. There were two obvious exceptions to this rule. First, scores on the BIQ performance situations subscale were less convincingly connected to various PAS-R scales. Second, symptoms of OCD as indexed by the PAS-R were only to some extent related to BIQ scales in 12-15year-olds but not in the younger age groups.

To examine whether behavioral inhibition as indexed by the BIQ is significantly stronger related to symptoms of social anxiety than to other anxiety symptoms, two statistical procedures were conducted. First, in all three age groups, tests were carried out to 
Table 3 Correlations between the parent-reported BIQ and PAS-R for the three age groups separately

\begin{tabular}{|c|c|c|c|c|c|c|}
\hline \multirow[t]{2}{*}{ BIQ } & \multicolumn{6}{|c|}{ PAS-R } \\
\hline & Total & Social & GAD & SAD & Fears & OCD \\
\hline \multicolumn{7}{|l|}{ 4-7-year-olds } \\
\hline Total score & $.74 *$ & $.83 *$ & $.58 *$ & $.51 *$ & $.38 *$ & .20 \\
\hline Peers & $.66^{*}$ & $.78 *$ & $.57 *$ & $.38 *$ & $.29^{*}$ & .20 \\
\hline Unfamiliar adults & $.56^{*}$ & $.73 *$ & $.34 *$ & $.36^{*}$ & $.30 *$ & .16 \\
\hline Performance situations & $.41^{*}$ & $.58 *$ & $.28 *$ & .22 & .17 & .05 \\
\hline Preschool/separation & $.66^{*}$ & $.66^{*}$ & $.55^{*}$ & $.55^{*}$ & $.35^{*}$ & .16 \\
\hline Unfamiliar situations & $.71^{*}$ & $.77 *$ & $.58^{*}$ & $.52 *$ & $.37 *$ & .23 \\
\hline Physical challenges & $.41^{*}$ & $.27 *$ & $.35^{*}$ & $.37 *$ & $.37 *$ & .12 \\
\hline \multicolumn{7}{|l|}{ 8-11-year-olds } \\
\hline Total score & $.66^{*}$ & $.75 *$ & $.56^{*}$ & $.42 *$ & $.33^{*}$ & .21 \\
\hline Peers & $.63^{*}$ & $.73 *$ & $.50 *$ & $.36^{*}$ & $.35^{*}$ & .25 \\
\hline Unfamiliar adults & $.47^{*}$ & $.59 *$ & $.38 *$ & $.35^{*}$ & .18 & .07 \\
\hline Performance situations & .17 & $.36 *$ & .07 & .09 & -.00 & -.05 \\
\hline Preschool/separation & $.66^{*}$ & $.65^{*}$ & $.61 *$ & $.48^{*}$ & $.34^{*}$ & .27 \\
\hline Unfamiliar situations & $.63^{*}$ & $.68 *$ & $.56^{*}$ & $.40 *$ & $.31 *$ & .21 \\
\hline Physical challenges & $.51 *$ & $.43^{*}$ & $.47 *$ & .23 & $.43^{*}$ & .28 \\
\hline \multicolumn{7}{|l|}{ 12-15-year-olds } \\
\hline Total score & $.66^{*}$ & $.77 *$ & $.54 *$ & $.42 *$ & $.37^{*}$ & $.31 *$ \\
\hline Peers & $.57 *$ & $.76^{*}$ & $.44^{*}$ & $.36^{*}$ & $.27^{*}$ & .23 \\
\hline Unfamiliar adults & $.45^{*}$ & $.62 *$ & $.34 *$ & .21 & .23 & .15 \\
\hline Performance situations & .23 & $.42 *$ & .20 & .07 & .00 & .02 \\
\hline Preschool/separation & $.70 *$ & $.65 *$ & $.59 *$ & $.54 *$ & $.51 *$ & $.43^{*}$ \\
\hline Unfamiliar situations & $.67 *$ & $.70 *$ & $.59 *$ & $.49^{*}$ & $.40^{*}$ & $.36^{*}$ \\
\hline Physical challenges & $.50 *$ & $.45^{*}$ & $.41 *$ & $.33^{*}$ & $.41 *$ & $.32 *$ \\
\hline
\end{tabular}

$n(4-7$-year-olds $)=172 ; n(8-11$-year-olds $)=146 ; n(12-15$-year-olds $)=213, B I Q$ Behavioral Inhibition Questionnaire, PAS-R Preschool Anxiety Scale-Revised, Total PAS-R total scale, Social PAS-R social anxiety, GAD PAS-R generalized anxiety, $S A D$ PAS-R separation anxiety, Fears PAS-R specific fears, $O C D$ PAS-R obsessive-compulsive disorder

$* p<.001$

compare the correlations [24] between BIQ (sub)scales and PAS-R social anxiety and the most substantial correlations between BIQ (sub)scales and another type of PAS-R anxiety symptoms. These analyses indicated that within each age group the BIQ total score correlated significantly stronger with PAS-R social anxiety than with PAS-R generalized anxiety, which was the second type of anxiety symptoms that correlated most substantially with this scale (4-7-year-olds: $Z=6.71, p<.001 ; 8-11$-year-olds: $Z=4.19, p<.001$; $12-15$-year-olds: $Z=5.80, p<.001)$. A similar pattern was observed for the BIQ subscales (all $Z s \geq 2.44, p<.05$ ), except for BIQ physical challenges (all age groups) and BIQ preschool/separation subscales (8-11-year-olds and 12-15-year-olds). Thus, BIQ subscale scores were in general more convincingly related to symptoms of social anxiety than to other anxiety symptoms.

Second, a series of stepwise regression analyses was conducted to examine the relative contributions of the PAS-R social anxiety subscale and the subscales measuring other types 
of anxiety symptoms to various BIQ scales. In all three age groups, PAS-R social anxiety turned out to be the only significant predictor of most BIQ scales, accounting for between 7 and $68 \%$ of the variance (all $F \mathrm{~s} \geq 13.19, p<.001$ ). For the non-social (preschool/separation and unfamiliar situations) and physical challenges subscales, other types of anxiety symptoms were also found to explain a significant proportion of the variance (all $F \mathrm{~s} \geq 8.71, p \mathrm{~s}<.001$; all $R^{2}$ change values between .01 and .14). For the non-social subscales PAS-R social anxiety still made the largest contribution to the model, but for the physical challenges subscale other types of anxiety symptoms were the main predictors. That is, in 4-7-year-olds PAS-R specific fears $(\beta=.24, p<.001)$ was the only significant predictor of BIQ physical challenges scores. In 8-11-year-olds PAS-R generalized anxiety $(\beta=.33, p<.01)$ and specific fears $(\beta=.27, p<.01)$ were found to make a significant contribution, whereas in $12-15$-year-olds PAS-R social anxiety $(B=.30, p<.001)$ and specific fears $(B=.26, p<.01)$ turned out to be significant predictors of this BIQ component.

\section{Parent-Child Agreement}

Table 4 displays mean scores (and SD) on the parent-rated and self-report version of the $\mathrm{BIQ}$, the internal consistencies of the child version, and the correlations between parentand child-rated behavioral inhibition. Note that the internal consistency of the self-report version of the BIQ was somewhat lower than that of the parent version (see Table 2) but generally still in an acceptable range (i.e., Cronbach's alphas varied between .65 and .91). Only the physical challenges subscale had an internal consistency that was too low $($ Cronbach's alpha $=.49)$. Further, on some BIQ subscales children rated themselves as (slightly) more inhibited than their parents did. More precisely, paired $t$-tests revealed that children scored higher on BIQ scales referring to unfamiliar adults $[t(292)=2.98$, $p<.01$, partial $\eta^{2}=.08$ ], preschool/separation $\left[t(292)=3.05, p<.01\right.$, partial $\eta^{2}=.06$ ], and unfamiliar situations $\left[t(289)=2.30, p<.05\right.$, partial $\left.\eta^{2}=.07\right]$. Finally, correlations between parent- and child-rated behavioral inhibition were moderate to high and varied

Table 4 Comparison of the mean scores of the parents and children on and correlations between the parent and children's scores

\begin{tabular}{lllll}
\hline Scale & Parents & \multicolumn{2}{l}{ Children (9-15-year-olds) } & \multirow{2}{*}{ Parent/child $r$} \\
& $M(\mathrm{SD})$ & $M(\mathrm{SD})$ & $\alpha$ & \\
\hline BIQ & & & & \\
Total score & $86.22(23.98)$ & $88.61(23.40)$ & .91 & $.59^{*}$ \\
Peers & $18.35(6.04)$ & $18.47(5.62)$ & .72 & $.45^{*}$ \\
Unfamiliar adults & $11.72(4.80)_{\mathrm{a}}$ & $12.57(5.03)_{\mathrm{b}}$ & .83 & $.50^{*}$ \\
Performance situations & $13.49(5.11)$ & $13.16(4.69)$ & .71 & $.51^{*}$ \\
Preschool/separation & $9.65(3.55)_{\mathrm{a}}$ & $10.35(3.65)_{\mathrm{b}}$ & .65 & $.40^{*}$ \\
Unfamiliar situations & $23.43(7.00)_{\mathrm{a}}$ & $24.37(7.53)_{\mathrm{b}}$ & .82 & $.55^{*}$ \\
Physical challenges & $9.68(3.90)$ & $9.85(3.70)$ & .49 & $.43 *$ \\
\hline
\end{tabular}

$n=293$. Means with different subscripts differ at $p<.05$ after Bonferroni correction

$B I Q$ Behavioral Inhibition Questionnaire

$* p<.001$ 
between .39 (BIQ preschool/separation) and .59 (BIQ total score). This agreement between parents and children did not vary as a function of age. That is, comparable results were found when computing separate parent-child correlations for youths aged 9 to 11 years ( $r$ s between .32 and .59 ) and 12 to 15 years ( $r$ s between .34 and .58 ).

\section{Discussion}

The present study examined the psychometric properties of the BIQ in a community sample of Dutch children and adolescents with a broad age range (i.e., 4 to 15 years). In general, the results were in line with findings of previous research [13,14] and indicate that the BIQ has good reliability and validity and that this is not only the case in preschoolers but also in older youths.

Exploratory factor analysis of the BIQ yielded support for the six-factor model as suggested by Bishop et al. [13] and Edwards [14], and this appeared true in all three age groups. The items describing inhibited behaviors in relation to peers, preschool/separation, adults, performance situations, and physical challenges nicely clustered into separate components. The only exception was the unfamiliar situations factor which did not emerge clearly in this study, with many of its items loading more convincingly on other BIQ factors. This has probably to do with the fact that items of the BIQ unfamiliar situations subscale are formulated in a more general way. That is, most of these items pertain to "unknown people" or "new situations and activities", which likely cover the specific situations as described in the items of other BIQ subscales. For instance, the "unknown people" as described in the BIQ unfamiliar situations subscale probably include the "adult strangers", "new adult guests", "unfamiliar children", and "new children" of the BIQ adults and peers subscales. In a similar vein, the "new situations and activities" as referred to in items of the BIQ unfamiliar situations subscale also involve more specific novel experiences as described in the BIQ preschool/separation (i.e., adjustment to new situations) and physical challenge (i.e., exploration of new play equipment) subscales. Thus, it remains unclear to what extent the BIQ unfamiliar situations subscale really represents an independent component of behavioral inhibition. Confirmatory factor analysis on the other hand yielded a reasonable fit for the six correlated factors model as proposed by Bishop et al. [13], and this appeared not only true in the total sample, but also in the three age groups separately. Altogether, the conclusion seems warranted that the BIQ [13] adequately covers different social and non-social aspects of children's inhibited temperament.

The internal consistency of the BIQ was satisfactory, and this was generally also true for the child version that we administered to children aged 9 years and above. It should be mentioned, however, that the reliability coefficient of the physical challenges subscale of the self-report version was below acceptable limits (Cronbach's alpha $=.49$ ). This finding is not very surprising given the fact that this BIQ subscale only consists of four items referring to situations that do not occur very often (e.g., physical challenges, new play equipment). Future studies should further examine the reliability of the physical challenge subscale, and such investigation should also address the test-retest stability of this and other self-report BIQ scales.

Only minor age effects were found for the BIQ on the performance situations and physical challenges subscale. Especially the effect on the performance situations subscale was as expected, with the oldest children (i.e., 12-15-year-olds) exhibiting the highest levels of inhibited responses in performance situations such as singing and dancing in front of a group. Clearly, these results mimic the age patterns as observed for social anxiety in 
children [23]. However, no support was found for the suggestion that younger children would score higher on non-social aspects of behavioral inhibition, whereas older children would score higher on scales representing the social components of this temperamental trait $[1,21]$. On the contrary, an age trend was observed showing that older children (i.e., 12-15-year-olds) displayed higher inhibition scores in physical challenge situations (which can be considered as a non-social aspect of behavioral inhibition) than children in the younger age groups. In the meantime, it should be noted that this result is nicely in keeping with the observation in anxiety research that fears of bodily injury and physical danger typically arise during middle childhood [25]. It is possible that the upcoming of these fears makes children more cautious and inhibited in situations involving physical challenges, and as such may explain the age trend as found for this BIQ subscale.

In the current study there were few gender differences in behavioral inhibition, which is well in line with previous research [14, 26]. However, some authors have suggested that gender differences in behavioral inhibition depend on the context in which the trait is assessed. For example, Kochanska [27] observed that boys were more inhibited in new environments, whereas girls primarily showed such behaviors in response to new persons. The gender difference as found in the present study, namely that boys scored higher on the BIQ performance situations scale than girls is not in keeping with this observation [14]. However, the finding that boys are more reluctant to dance and sing in front of others is not that surprising. When looking at children on a schoolyard we often see groups of girls dancing and singing, while boys are playing football or engage in other physical activities [28]. Further, Ryan [29] observed significantly more anxious behaviors in boys prior to and during a performance situation (i.e., a musical performance) than in girls. Thus, although gender differences in behavioral inhibition and social anxiety seem to be small, future research is needed to further explore this issue.

This study also provides support for the validity of the BIQ in younger as well as older children. That is, substantial and positive correlations were found between BIQ total and subscale scores and scores on the PAS-R, a questionnaire for measuring DSM-defined anxiety symptoms, and this appeared true in children of all ages. Although BIQ scales were significantly associated with a broad range of anxiety symptoms, the data also indicated that social anxiety was most convincingly related to social as well as non-social aspects of this temperamental trait. Obviously, these findings are in keeping with the idea that behavioral inhibition is more important as a specific risk factor for social phobia than for other types of anxiety disorders [6, 8]. Surprisingly, even the non-social BIQ scales correlated most strongly with symptoms of social anxiety. One explanation for this unexpected result may be that these scales, although they intended to measure the non-social aspects of behavioral inhibition, still refer to social aspects of the inhibited temperament. For instance, items of the non-social BIQ scales such as "Is clingy when we visit homes of people we don't know well", "Is outgoing", and "Gets upset at being left in new situations for the first time (e.g., kindergarten, preschool, child care)" do not merely reflect nonsocial events but clearly incorporate social elements. The only real exception to this rule was the physical challenge subscale, which only contained items of a non-social nature. Interestingly, it was this particular BIQ scale that correlated equally strong with social anxiety and other anxiety symptoms. Thus, it seems that the strong correlation between non-social inhibition and social anxiety can be partly explained by the way non-social inhibition is measured.

Moderate to high correlations were found between parent- and child-rated inhibition. In addition, children rated themselves as slightly more inhibited than their parents did. On first sight, parent-child correlations in the .40 and .50 range do not seem that substantial, 
but these figures compare rather favorable to those obtained in previous studies on child characteristics [30, 31]. For example, Achenbach et al. [30] conducted a meta-analysis on the cross-informant agreement of various psychopathology questionnaires, and found a mean parent-child correlation of .25. Moreover, parent-child correlations for internalizing phenomena, like behavioral inhibition and anxiety, are generally even lower than those found for externalizing characteristics [31]. Thus, the parent-child agreement as reported in this study can be considered as quite good.

Several limitations of the present study should be noted. A first limitation pertains to the relatively low response percentage of the parents (approximately 23\%) who participated in the study. Most participants were Caucasian, and although not adequately documented, migrant parents and children were relatively underrepresented. Thus, research in populations that contain children and adolescents from various ethnic groups is required and this is of particular interest as data have shown that ethnic minority groups in the Netherlands report higher levels of anxiety disorder symptoms [32] and hence may also display greater vulnerability to develop such problems. Second, this study relied on normal, psychologically healthy youths, and so the reliability and validity of the BIQ in clinically referred youths remains to be established. Third, a number of psychometric properties of the BIQ remain to be tested. As mentioned earlier, the current study did not examine the test-retest reliability of the BIQ, and also the predictive and discriminant validity of the scale need to be investigated. In spite of these limitations, the present findings are encouraging as they yield first evidence for the reliability and validity of the BIQ as an index for measuring various aspects behavioral inhibition in youths with a broad age range.

\section{Summary}

This study provides preliminary support for the reliability and validity of the BIQ in a Dutch non-clinical sample including children and adolescents. In all age groups the internal consistency of most BIQ scales was found to be satisfactory. Exploratory factor analysis yielded a multiple-factor model that was largely in keeping with the hypothesized structure consisting of the social and non-social components of behavioral inhibition, and a CFA demonstrated reasonable fit for a six correlated factors model in the total sample as well as in the three age groups separately. Further, BIQ scores were positively correlated with a wide range of anxiety symptoms, although the most substantial links were found for symptoms of social anxiety. Finally, a self-report version of the BIQ, which was administered to children aged 9 years and above, also possessed good internal consistency and adequate parent-child agreement. Therefore, it can be concluded that the BIQ seems to be a promising scale for assessing various aspects of behavioral inhibition that is not only suitable for preschoolers but also for older children and adolescents.

Acknowledgments Parents, children and teachers from primary schools "Ds. J. J. Buskesschool" and "Tuinstad" in Rotterdam and "Gideonschool" in Nieuwerkerk aan den IJssel, and secondary schools "Comenius College" in Nieuwerkerk aan den IJssel and "Ostrea Lyceum" in Goes are kindly thanked for their participation in the present study. Lotte Timmerman is kindly acknowledged for her assistance with the data collection.

Open Access This article is distributed under the terms of the Creative Commons Attribution Noncommercial License which permits any noncommercial use, distribution, and reproduction in any medium, provided the original author(s) and source are credited. 


\section{References}

1. Hirshfeld-Becker DR, Biederman J, Rosenbaum JF (2004) Behavioral inhibition. In: Morris TL, March JS (eds) Anxiety disorders in children and adolescents. Guilford Press, New York, pp 27-58

2. Robinson JL, Kagan J, Reznick JS, Corley R (1992) The heritability of inhibited and uninhibited behavior: a twin study. Dev Psychol 28:1030-1037

3. Kagan J, Reznick JS, Clarke C, Snidman N, Garcia-Coll C (1984) Behavioral inhibition to the unfamiliar. Child Dev 55:2212-2225

4. Biederman J, Rosenbaum JF, Hirshfeld-Becker DR, Faraone SV, Bolduc EA, Gersten M, Meminger SR, Kagan J, Snidman N, Reznick JS (1990) Psychiatric correlates of behavioral inhibition in young children of parents with and without psychiatric disorders. Arch Gen Psychiatry 47:21-26

5. Biederman J, Rosenbaum JF, Bolduc-Murphy EA, Faraone SV, Chaloff J, Hirshfeld-Becker DR, Kagan J (1993) A 3-year follow-up of children with and without behavioral inhibition. J Am Acad Child Adolesc Psychiatry 32:814-821

6. Mick MA, Telch MJ (1998) Social anxiety and history of behavioral inhibition in young adults. J Anx Disord 12:1-20

7. Gladstone GL, Parker GB, Mitchell PB, Wilhelm KA, Malhi GS (2005) Relationship between selfreported childhood behavioral inhibition and lifetime anxiety disorders in a clinical sample. Depress Anx 22:103-113

8. Van Brakel AML, Muris P, Arntz A, Schouten E (2009) Behavioral inhibition as a risk factor for the development of childhood anxiety disorders: a longitudinal study (submitted)

9. Muris P, Meesters C, Spinder M (2003) Relationships between child- and parent-reported behavioural inhibition and symptoms of anxiety and depression in normal adolescents. Pers Individ Dif 34:759-771

10. Gest SD (1997) Behavioral inhibition: stability and associations with adaptation from childhood to early adulthood. J Pers Soc Psychol 72:467-475

11. Reznick JS, Hegeman JM, Kaufman ER, Woods SW, Jacobs M (1992) Retrospective and concurrent selfreport of behavioral inhibition and their relation to adult mental health. Dev Psychopathol 4:301-321

12. Van Brakel AML, Muris P, Bögels SM (2001) Gedragsinhibitie als risicofactor voor het ontwikkelen van angststoornissen bij kinderen: Een overzicht (Behavioral inhibition as a risk factor for the development of anxiety disorders in children: a review). Nederlands Tijdschrift voor de Psychologie en haar grensgebieden 56:57-68

13. Bishop G, Spence SH, McDonald C (2003) Can parents and teachers provide a reliable and valid report of behavioral inhibition? Child Dev 74:1899-1917

14. Edwards SL (2007) Temperament and environmental risk factors contributing to anxiety symptoms in preschool-aged children. Unpublished thesis, Macquarie University, Sydney, Australia

15. Kagan J, Reznick JS, Snidman N (1988) Biological bases of childhood shyness. Science 240:167-171

16. Scarpa A, Raine A, Venables PH, Mednick SA (1995) The stability of inhibited/uninhibited temperament from ages 3 to 11 years in Mauritian children. J Abnorm Child Psychol 23:607-618

17. Fox NA, Henderson HA, Marshall PJ, Nichols KE, Ghera MM (2005) Behavioral inhibition: linking biology and behavior within a developmental framework. Ann Rev Psychol 56:235-262

18. Spence SH, Rapee R, McDonald C, Ingram M (2001) The structure of anxiety symptoms among preschoolers. Behav Res Ther 39:1293-1316

19. American Psychiatric Association (2000) Diagnostic and statistical manual of mental disorders, 4th edn. American Psychiatric Association, Washington, DC

20. Achenbach TM (1991) Manual for the child behavior checklist 4-18 and 1991 Profile. University of Vermont, Department of Psychiatry, Burlington

21. Broeren S, Muris P (2008) Psychometric evaluation of two new parent-rating scales for measuring anxiety symptoms in young Dutch children. J Anx Disord 22:949-958

22. Byrne BM (2001) Structural equation modeling with AMOS: basic concepts, applications, and programming. Lawrence Erlbaum, Mahwah

23. Hu L, Bentler PM (1999) Cutoff criteria for fit indexes in covariance structure analysis: conventional criteria versus new alternatives. Struct Equ Modeling 6:1-55

24. Meng X, Rosenthal R, Rubin DB (1992) Comparing correlated correlation coefficients. Psychol Bull 111:172-175

25. Bauer DH (1976) An exploratory study of developmental changes in children's fears. J Child Psychol Psychiatry 17:69-74

26. Garcia-Coll C, Kagan J, Reznick JS (1984) Behavioral inhibition in young children. Child Dev 55:1005-1019

27. Kochanska G (1991) Patterns of inhibition to the unfamiliar in children of normal and affectively ill mothers. Child Dev 62:250-263 
28. Thorne B (1993) Gender play: girls and boys in school. Academic Internet Publishers, New Brunswick

29. Ryan C (2004) Gender differences in children's experience of musical performance anxiety. Psychol Music 32:89-103

30. Achenbach TM, McConaughy SH, Howell CT (1987) Child/adolescent behavioral and emotional problems: implications of cross-informant correlations for situational specificity. Psychol Bull 101:213-232

31. Kolko DJ, Kazdin AE (1993) Emotional/behavioral problems in clinic and nonclinic children: correspondence among child, parent and teacher reports. J Child Psychol Psychiatry 34:991-1006

32. Hale WW III, Raaijmakers Q, Muris P, Meeus W (2005) Psychometric properties of the Screen for Child Anxiety Related Emotional Disorders (SCARED) in the general adolescent population. J Am Acad Child Adolesc Psychiatry 44:283-290 